\title{
Modification of The Rheological Properties of Asphalt Using Spent Lubricating Oils and Air Oxidation
}

\author{
Alaa A. Hussein \\ Department of Environmental Sciences \\ College of Environmental Sciences and \\ Technology
}

Ammar A. Hamdoon

\author{
Department of Chemistry \\ College of Education for Pure Science \\ ammarhamdoon@uomosul.edu.iq
}

$\begin{array}{cc}\text { Received } & \text { Accepted } \\ \mathbf{0 3 / 0 5 / 2 0 1 8} & \mathbf{0 7 / 0 6 / 2 0 1 8}\end{array}$

\begin{abstract}
There is a growing need for the production of certain types of asphaltic materials having good rheological properties compared with the unmodified asphaltic materials, thus many experiments were performed to prepare a modified asphalt that can be used in different fields. This study aims to modify asphalt with the addition of (spent lubricating oils) with different percentages and with air blowing oxidation.

From this work we obtained the asphaltic materials which can be used in paving and mastic production according to the measuring of the (ductility, penetration, asphaltene and softening point)
\end{abstract}

keywords: Asphalt, Rheological Properties, Polymeric Additives, Asphalt Oxidation 


\section{تحوير الخواص الريولوجية للإسفلت باستعمال الزيوت المستهلكة والأكسدة الهوائية}

\author{
عمار احمد حمدون \\ قسم الكيمياء \\ كلية التربية للعلوم الصرفة

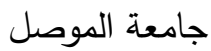

ammarhamdoon@uomosul.edu.iq

\author{
آلاء علي حسين \\ قسم علوم البيئة \\ كلية علوم البيئة وتقاناتها

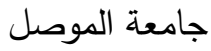

\section{تاريخ الاستلام ت ت \\ 2018/06/07 2018/05/03}

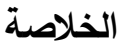

نظراً للحاجة الماسة لإنتاج مواد ذات جدوى اقتصادية كبيرة تتمثل في إنتاج مواد إسفلتية ذات مواصفات ريولوجية تختلف عن مواصفات استعمال الإسفلت الاعتيادي اشتملت هذه الدراسة على معاملة الإسفلت مع الزيوت بنسب مختلفة وبوجود عملية أكسدة هوائية .

تم الحصول من خلال هذه الدراسة على نماذج إسفلتية ذات مواصفات ريولوجية بالإمكان استعمالها في مجال التبليط وأخرى يمكن استعمالها كمواد مانعة للرطوبة اعتماداً على القياسات التي تم إجراؤها (الاستطالة ، النفاذية ، درجة الليونة ، نسبة الاسفلتين ، حساب دليل الاختراق).

الكلمات المفتاحية: الإسفلت ، الخواص الريولوجية ، المضافات البوليمرية ، أكسدة الإسفلت

\section{المقدمة}

الإسفلت عبارة عن مادة ذات لون اسود أو بني غامق غروية شبه صلبة وتكون ذات لزوجة عالية في درجة حرارة الغرفة ويتم الحصول عليه بوساطة عمليات التقطير المختلفة للنفط الخام [2,1]. والقير عموماً هي المادة الأكثر استعمالاً في التبليط بسبب أداء خدمته المتميزة من حيث الثبات والتحمل ومقاومته للماء [3] . وان الخمول الكيميائي الذي يمتاز به الإسفلت والمتوافق مع خواصه الفيزيائية هو الأمر الذي يجعله مؤهلاً لاستعمالات واسعة في الصناعة والبناء منذ الازل وان طبيعة الصفات الفيزيائية له هي التي تحدد طبيعة الاستعمال [5,4] ، ان عملية تحسين أداء الخلطات الإسفلتية يتم بطرائق مختلفة باستعمال المضافات ، وبالرجوع إلى الأدبيات نجد العديد من الدراسات التي تناولت عملية تحوير مواصفات الإسفلت بطرائق مختلفة ومنها ما قام به Thakre [6] حيث حور الصفات الريولوجية للاسفلت باضافة البوليمرات اثيلين فينايل اسيتايت(EVA) وفتات المطاط ، واعطت الدراسة نماذج اسفلتية اكثر مقاومة للإعياء ، التثقق الحراري ، التعفن Rutting ، والحساسية لدرجة الحرارة، بالاككان استعمالها في مجال التبليط. 
ودرس Maharaj \&Maharaj [7] الخواص الفيزيائية للإسفلت بإضافة البولي اثيلين واطئ الكثافة والبولي كلوريد الفاينيل وزيت المحركات المستهلك إذ بينت النتائج انه بازدياد تركيز زيت المحركات المستعمل بنسب (0-30) حصلت زيادة في صفة مقاومة تثققات الإجهاد ورافقه انخفاض في مقاومة تثكيل الأخاديد في الثارع.

وقام Dekhli وآخرون [8] بدراسة السلوك الريولوجي للاسفلت المحور بإضافة اثيلين فاينيل اسيتايت

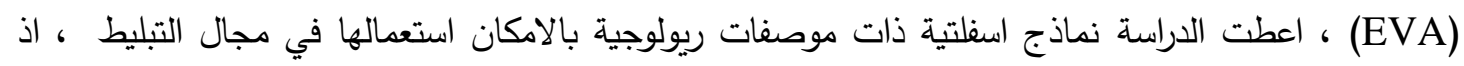
كان هناك تغير ايجابي واضح في القياسات ( درجة الليونة ، النفاذية ، الحساسية لدرجة الحرارة). وتمكن Djaffar و وآخرون [9] بدراسة الخواص الريولوجية والثباتيةعند الخزن للاسفلت المحور بإضافة بوليمر ستايرين - اتلين - بيوتلين - ستايرين (SEBS) ، اذ اكدت الدراسة على ثبات استقرارية الخواص الريولوجية ( النفاذية ، درجة الليونة ، دليل الاختراق ، الحساسية لدرجة الحرارة) للإسفلت المحور من خلال قياسها بعد الخزن لفترة زمنية. ودرس Al-Zubaidi [10] تحوير الخواص الريولوجية للإسفلت بإضافة الزيت المستهلك بإسلوبين الأول

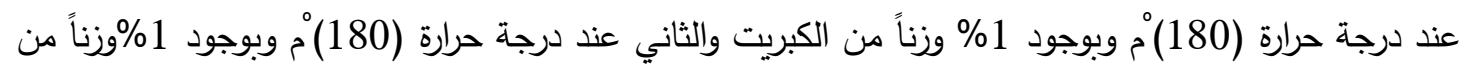
كلوريد الأمنيوم اللامائي و1\% وزناً من الكبريت وفي كلا المسارين تم الحصول على نماذج معظمها ذات مواصفات ريولوجية جيدة.

واستعمل Can [11] الزيت المستهلك بنسب مختلفة تراوحت بين (1- 12)\% اضيف الى الاسفلت بدرجات حرارة منخفضة واعطت دراسته نتائج جيدة في تقليل انتزاع او قثط الاسفلت. وقام Arslan \& Taque [12] بدراسة الخواص الريولوجية للاسفلت بمعاملته مع نسب مختلفة من زيت التزييت بالتسخين وكان هناك تغير ايجابي واضح في القياسات من (درجة الليونة ، والنفاذية ، وقابلية المط والفقدان بالوزن).

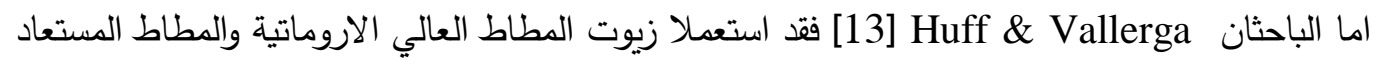
من اطار السيارات كمادة مضافة الى الاسفلت واستغلت النماذج المطورة في تبليط الطرق والسطوح المقاومة للماء. وتمكن Al-Sayd Toohi [14] من دراسة تأثير نظام (مطاط _ كبريت) على الخواص الريولوجية للإسفلت بإسلوبين،الأول يعتمد على تحوير الإسفلت مع المطاط المعاد باستعمال نسب مختلفة من حفاز كلوريد الألمنيوم اللامائي عند طاقات مختلفة من اشعة المايكرويف وبأزمان مختلفة والأسلوب الثاني يعتمد على المبدأ نفسه مع إدخال الكبريت كمادة إضافية وبنسب مختلفة وتم الحصول من المسارين على نماذج إسفلتية ذات مواصفات ريولوجية بالإمكان استعمالها في مجال التبليط وأخرى يمكن استعمالها كمواد مانعة للرطوبة. اما الباحثان Sadeque \& Patil [15 استخدما البولي اثثلين واطئ الكثافة (LDPE) والبولي بروبلين(PP) كمادة مضافة للإسفلت عند (200)ْ اذ لاحظوا تحسن واضح للخواص الريولوجية للإسفلت (النفاذية ، درجة الليونة ، الاستطالة ). 
وقام Bulatovic وآخرون [16] بدراسة الصفات الريولوجية لإسفلت الطرق بإضافة (ستايرينبيوتادايين - ستايرين) اذ حسنت الدراسة بصورة ملحوظة الخواص لإسفلت الطرق (النفاذية ، درجة الليونة ، دليل الاختراق).

واستعملت Al-Liheby [17 نوع من اللواصق والكبريت كمضافات للإسفلت إذ تمكنت من إنتاج أنظمة إسفلتية جديدة ملائمة للاستعمال في مجال التبليط. كما تمكن الباحثان Anjan \& Veeragavan [18 من مقارنة الاسفلت الاصلي والاسفلت المحسن من خلال خلط الاسفلت بالمضافات لتحسين الخواص الميكانيكية للاسفلت واشارت النتائج الى اختلاف كبير بين الاسفلت الاصلي والمحور . ودرست Salih [19] تأثير النفخ بالهواء على استقرارية مزائج (اسفلت قيارة - كبريت) بإسلوبين الاول مزج اسفلت مؤكسد مع نسب مختلفة من الكبريت اما الاسلوب الثاني اجراء تفاعل كيميائي مابين الاسفلت المؤكسد والكبريت قبل إضافة النسب المحددة من الكبريت حيث اظهرت نتائج القياسات والفحص المايكروسكوبي تحسن في تجانس النظام الاسفلتي مقارنة بالمزائج المحضرة من الاسفلت غير المحور.

\section{الجزء العملي}

1- الأكسدة الهوائية للمادة الإسفلتية

اخذ وزن معين من الإسفلت والذي تم الحصول عليه من شركة نفط الثمال والذي يمتاز بالمواصفات

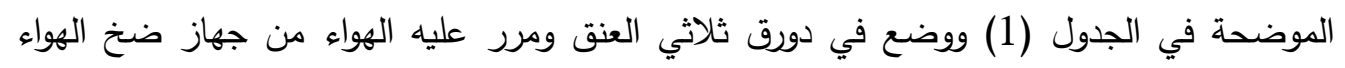

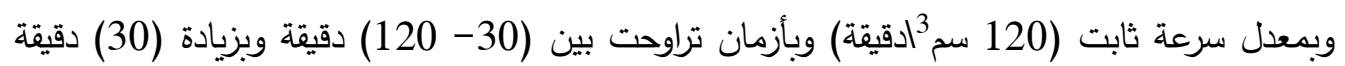

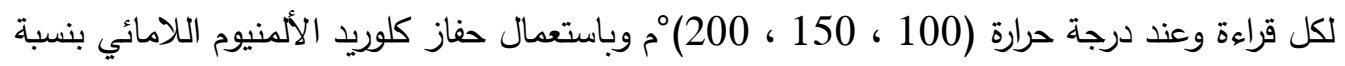
.

الجدول (1): الخواص الريولوجية للمادة الإسفلتية

\begin{tabular}{|c|c|}
\hline القيمة المختبريـة & المواصفات \\
\hline 48 & درجة الليونة (م) \\
\hline 59 & النفاذية ، ملم (100 غم ، 5ثا ، 25 مْ) \\
\hline $100+$ & الاستطالة (سم ، 25م) \\
\hline
\end{tabular}

2- تحديد النسبة المثلى لحفاز كلوريد الألمنيوم اللامائي

بعد تحديد درجة الحرارة المثلى لعملية الأكسدة تم تغير نسبة الحفاز المستعملة ، اذ استعملت النسب

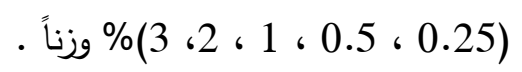
3- - معاملة الإسفلت مع الزيت المستهلك 
عومل الإسفلت مع نسب مختلفة من الزيت المستهلك بنسب تراوحت بين (1-4)\% وزناً وبزيادة 1\%

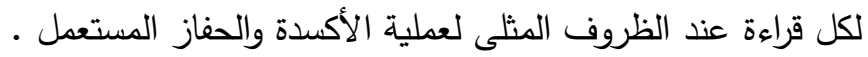

\section{4- فصل الاسفلتين}

جميع النماذج المحضرة اجريت عليها عملية فصل للاسفلتين بالاعتماد على الطرائق القياسية [20].

\section{5- تحديد مواصفات المواد الإسفلتية الأصل والمعاملة}

تم تحديد المواصفات للمواد الإسفلتية الأصل والمعاملة وشمل ذلك قياس كل من النفاذية [21] والاستطالة [22] ودرجة الليونة [23] وحساب دليل الاختراق ( PI ) [24] لجميع النماذج •

\section{النتائج والمناقشة}

ان تفاعلات الأكسدة مهمة جداً في مجال الكيمياء الصناعية وقد استعملت ابتداءً من بضع كيلو غرامات إلى ألاف الأطنان سنوياً . وفي عمليات الأكسدة الصناعية التي تجري على نطاق ضخم يُتَجَنب فيها استعمال

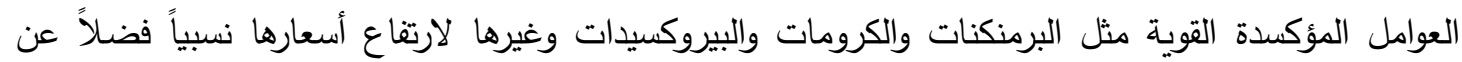
خطورتها بسبب صعوبة السيطرة على تفاعلات الأكسدة الباعثة للحرارة لذلك فان هناك حاجة ملحة لاستعمال

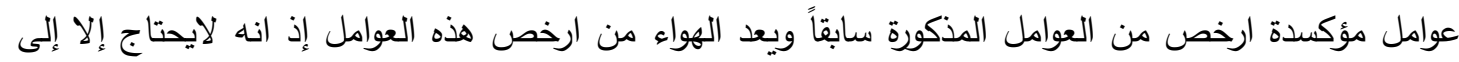
تكاليف قليلة تتضمن كيفية التعامل معهد في العديد من المشروعات الكبيرة . اجريت عملية الأكسدة وكما هو موضح في الجزء العملي والجدول (2) يوضح النتائج التي تم الحصول

الجدول (2): الخواص الريولوجية للإسفلت المؤكسد هوائياً عند درجات حرارية وأزمان مختلفة وبوجود 0.5\%

\begin{tabular}{|c|c|c|c|c|c|c|c|}
\hline دليل الاختراق & درجة الليونة & النفاذية & الاستطالة & الاسفلتين(\%) & الحرارة(م) دمجة & الزمن & النموذج \\
\hline-1.332 & 48 & 59 & $100+$ & 23 & ------ & $\begin{array}{l}--- \\
\end{array}$ & $\mathrm{AS}_{0}$ \\
\hline-1.052 & 53 & 38.5 & 67 & 30 & 100 & 30 & $\mathrm{AS}_{1}$ \\
\hline-0.696 & 54 & 41 & 70 & 31 & 100 & 60 & $\mathrm{AS}_{2}$ \\
\hline-0.424 & 55 & 42 & 76 & 32 & 100 & 90 & $\mathrm{AS}_{3}$ \\
\hline-0.508 & 56 & 36.6 & 43 & 31 & 100 & 120 & $\mathrm{AS}_{4}$ \\
\hline-0.787 & 54 & 39.3 & 94 & 25 & 150 & 30 & $\mathrm{AS}_{5}$ \\
\hline-0.477 & 55 & 41 & $100+$ & 27 & 150 & 60 & $\mathrm{AS}_{6}$ \\
\hline-0.484 & 56 & 37 & $100+$ & 37 & 150 & 90 & $\mathrm{AS}_{7}$ \\
\hline-0.395 & 57 & 35 & 88 & 39 & 150 & 120 & $\mathrm{AS}_{8}$ \\
\hline-0.601 & 56 & 35 & 77 & 34 & 200 & 30 & $\mathrm{AS}_{9}$ \\
\hline-0.912 & 54 & 37 & 85 & 35 & 200 & 60 & $\mathrm{AS}_{10}$ \\
\hline 0.096 & 58 & 40 & 62 & 36 & 200 & 90 & $\mathrm{AS}_{11}$ \\
\hline-0.811 & 55 & 35 & $100^{+}$ & 30 & 200 & 120 & $\mathrm{AS}_{12}$ \\
\hline
\end{tabular}


يتضح من الجدول أعلاه إن أفضل النماذج تم الحصول عليها باستعمال ظروف أكسدة هوائية محفزة ب(0.5)\%وزناً من كلوريد الالمنيوم اللامائي عند (150ْم ، 60 دقيقة ، 120سم3/دقيقة) (درجة حرارة ، زمن

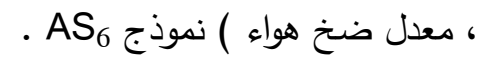

ان سير التفاعلات أثناء عملية الأكسدة بالاتجاه

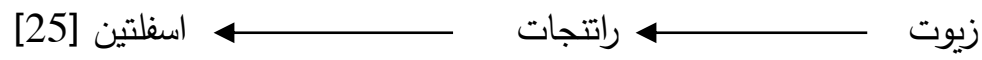

إذ يمكن القول ان الراتجات تمثل حلقة وصل بين الاسفلتينات ذات الأوزان الجزيئية الأعلى والزيوت ذات الأوزان الجزئية الأقل من الراتنجات إذ يمكن أكسدة الراتنجات بسهولة بوساطة الهواء الجوي في درجات الحرارة الاعتيادية حيث تتحول إلى اسفلتينات .والنتائج توضح سير هذه العملية من خلال متابعة نسبة الاسفلتين التي تم الحصول عليها من خلال عملية الأكسدة ـ ان الزيادة والنقصان في قيمة الاسفلتين المفصولة تعتمد بدرجة كبيرة على سرعة تفاعلات التكاثف التي تؤدي الى زيادة نسبة الاسفلتين او تفاعلات الانحلال التي تؤدي الى انخفاض لتصني نسبة الاسفلتين. بعد تثبيت درجة الحرارة وزمن الأكسدة قمنا بتحديد أفضل نسبة مستعملة من الحفاز والجدول (3) يوضح النتائج التي تم الحصول عليها .

الجدول (3): الخواص الريولوجية للإسفلت المعامل مع نسب مختلفة من كلوريد الألمنيوم اللامائي عند

(150)

\begin{tabular}{|c|c|c|c|c|c|c|}
\hline دليل الاختراق & الليونة & النفاذية & الاستطالة & الاسفلتين(\%) & الحفاز (\%بة) & النموذج \\
\hline-1.332 & 48 & 59 & $100+$ & 23 & ------- & $\mathrm{AS}_{0}$ \\
\hline-0.277 & 57 & 37 & 95 & 28.8 & 0.25 & $\mathrm{AS}_{13}$ \\
\hline-0.477 & 55 & 41 & $100+$ & 27 & 0.5 & $\mathrm{AS}_{14}$ \\
\hline-0.378 & 58 & 32 & 76 & 33.5 & 1 & $\mathrm{AS}_{15}$ \\
\hline-0.414 & 59 & 28.5 & 28 & 36.8 & 2 & $\mathrm{AS}_{16}$ \\
\hline-0.111 & 62 & 25 & 16 & 42 & 3 & $\mathrm{AS}_{17}$ \\
\hline
\end{tabular}

يتضح من الجدول أعلاه ان النسبة التي تم استعمالها من بدء الدراسة وبصورة افتراضية تمثل أفضل نسبة مستعملة من الحفاز والتي تعطي إسفلتاً ذا مواصفات ريولوجية جيدة . بعد تثبيت جميع الظروف أعلاه تم إضافة الزيت المستهلك كمادة مضافة إلى الإسفلت لمعرفة تأثيرها على دوالى الخواص الريولوجية للمادة الإسفلتية والجدول (4) يوضح النتائج التي تم الحصول عليها ـ 


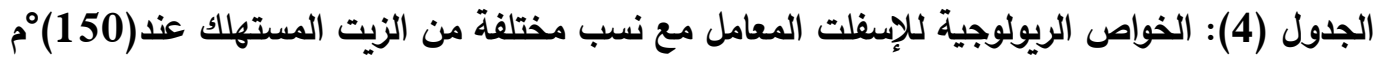

وزمن ساعة بوجود (0.5)\% وزناً من كلوريد الأمنيوم اللامائي بعملية أكسدة هوائية

\begin{tabular}{|c|c|c|c|c|c|c|}
\hline دليل الاختراق & الليونة & النفاذية & الاستطالة & الاسفلتين(\%) & الزيت(\%بة) & النموذج \\
\hline-1.332 & 48 & 59 & $100^{+}$ & 23 & ----- & $\mathrm{AS}_{0}$ \\
\hline-0.749 & 54 & 40 & 65 & 32 & 1 & $\mathrm{AS}_{18}$ \\
\hline-0.668 & 53 & 46 & 83 & 33 & 2 & $\mathrm{AS}_{19}$ \\
\hline-0.951 & 51 & 50 & $100+$ & 34 & 3 & $\mathrm{AS}_{20}$ \\
\hline-0.907 & 49 & 63 & 67 & 35 & 4 & $\mathrm{AS}_{21}$ \\
\hline
\end{tabular}

يتضح من الجدول أعلاه ان النموذج (AS $)$ كان أفضل نموذج من حيث مواصفاته المقاربة لمواصفات

اسفلت التبليط .

ان استعمال الزيت المستهلك كمادة مضافة يعدُ اختيارً جيداً إذ ان كل النماذج التي تم الحصول عليها

كانت تتصف بالتجانس لكون كل من المادة الأولية والمادة المضافة تتبع من أصل واحد أي (بترولية المنشأ) لذلك كان تأثيرها واضحاً في الحصول على نماذج جيدة . ويعتقد الباحثون ان عملية الأكسدة شملت عملية تهثيم بهيئة جذور حرة فعالة يليها عملية تكاثف من

خلال المجموعات أو الذرات الهجينة ويؤدي في النهاية إلى زيادة الوزن الجزيئي وزيادة نسبة الاسفلتين [26,1]

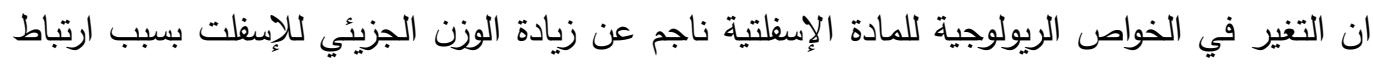

المضافات ذات الأوزان الجزيئية العالية بالتراكيب الإسفلتية عن طريق حدوث لإنيات لفاعلات كيميائية .

ان جميع النماذج المعاملة يمكن الاستفادة منها في مجالات عدة فهناك نماذج يمكن ان تستعمل في مجال التبليط وأخرى في مجال التسطيح وأخرى كمواد ماستك أما النماذج الرديئة وذات نسب الاسفلتين العالية

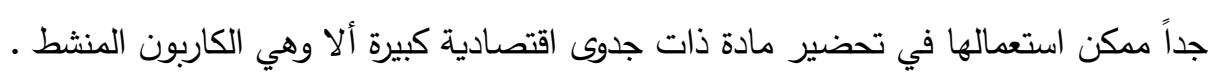
اما (Penetration Index-PI) فهو عبارة عن علاقة متطورة تربط بين درجة ليونة النموذج وبين درجة نفاذيته في (25) مْ ويتم التعرف من خلال هذه الدالة على مدى حساسية المادة الاسفلتية وتاثرها بدرجات الحرارة ، ويمكن حساب تلك الدالة عن طريق تعويض قيم درجات الليونة والنفاذية للنموذج الاسفلتي من العلاقة $=50\left(\frac{\log 800-\log P e n t}{T_{R . B}-T}\right) \frac{20-P I}{10+P I}$

الرياضية [27]. اذ ان • Pent • دليل الاختراق PI درجة ليونة النموذج المقاسة بطريقة الكرة والحلقة. 


$$
\text { د درجة الحرارة التي تم عندها قياس درجة النفاذية = (25) T م. }
$$

يمكن القول عموماً بان الانواع الاسفلتية الجيدة ذات المواصفات الملائمة للاستعمال في مجال التبليط

يجب ان تمتلك دليل اختراق ذا قيمة تقع بين (- 2 الى +2) [28] ـ بعد ملاحظة النتائج التي تم الحصول عليها في هذه الدراسة ومن خلال اجراء قياسات المواصفات الريولوجية للنماذج المحضرة نجد ان بعض تلك النماذج تميزت بمواصفات تؤهلها للاستعمال كاسفلت تبليط في حين امتلكت بعض النماذج مواصفات تؤهلها للاستعمال كماستك (Mastic) وهي مادة عازلة للرطوبة ويدخل في العديد من الاستعمالات ومنها مايستعمل لاغراض التسقيف والتسطيح وغيرها من الاستعمالات والجدول (7،6،5 توضح مواصفات الاسفلت المستعمل كماستك والاسفلت المستعمل في مجال التبليط والتسطيح .

الجدول (5): المواصفات القياسية الامريكية (41 - D491) للاسفلت المستعمل لانتاج الماستك [29].

\begin{tabular}{|c|c|c|}
\hline الحد الاعلى & الحد الادنى & القياسات الريولوجية \\
\hline 65 & 54 & درجة الليونة (مم) \\
\hline 40 & 20 & النفاذية ، ملم (100غم ، 5ثا ،25م) \\
\hline--- & 15 & الاستطالة (سم ، 25 م) \\
\hline
\end{tabular}

الجدول (6): المواصفات القياسية العراقية للاسفلت المستعمل في التسطيح [30] .

\begin{tabular}{|c|c|c|}
\hline الحد الاعلى & الحد الادنى & القياسات الريولوجية \\
\hline 66 & 57 & درجة الليونة (م) \\
\hline 40 & 18 & النفاذية ، ملم (100غم ، 5ثا ،25 م) \\
\hline--- & 10 & الاستطالة (سم ، 25॰ \\
\hline
\end{tabular}

الجدول (7): يبين الخواص الريولوجية للاسفلت المستعمل في التبليط حسب مواصفات هيئة الطرق والجسور

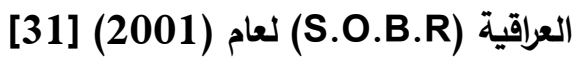

\begin{tabular}{|c|c|c|}
\hline الحد الاعلى & الحد الادنى & القياسات الريولوجية \\
\hline 60 & 54 & درجة الليونة (مم) \\
\hline 50 & 40 & النفاذية ، ملم (100غم ، 5ثا ،25م) \\
\hline--- & 100 & الاستطالة (سم ، 25 م) \\
\hline
\end{tabular}




\section{المصادر}

1- Parkash, S., "Petroleum Fuels Manufacturing Hand Book", Mc Graw-Hill Companies, Inc. 102-131, (2010).

2- Zhang, L. and Green field M.L, "Effects of Polymer Modification on Properties and Microstructure of Model asphalt Systems", Energy \& Fuels, Vol. 22, pp. 3363-3375,(2008).

3- Polacco, G. Stastna, J., Biondi, D. Antonelli, F., Vlachovicova, Z. and Zanzotto, L., "Rheology of asphalts modified with glycidylmethacrylate functionalized polymers". J. of colloid and interface science, Vol. 280, pp. 336-373, (2004).

4- Lesueur D., " The Colloidal Structure of Bitumen: Consequences on the Rheology and on the Mechanisms Modification", Advances in colloid and Interface, p.145,28-42, (2009).

5- Introduction To Asphalt", Asphalt institute manual series No. 5(MS-5)P. 2,911, 14,61, (2001)

6- Thakre,N., Mangrulkar,D., Janbandhu,M. and Saxena,J., "Polymer Modified Bitumen", IOSR Journal of Mechaical and Civil Enginering Vol.13, Issue 6Ver, (2016).

7- Maharaj, Rean and Maharaj, Chris, "Physical Properties of Low Density Polyethylene, Polyvinyl chloride and used Engine oil Modified Asphalt”, Progress in Rubber and Recycling Technology, Vol. 31. 3, (2015).

8- Dekhli, S., Mokhtar, K..A., Hammoum, F.and Bachir, D.S., "Rheological Behaviour of Ethylene-Vinyl-Acetate(EVA) Modified Road Bitumen", Journal of Applied Sciences Vol. 15, No.(3), pp 444-455, (2015).

9- Djaffar, S., Samy, D. and Khadidja, A.M., " Rheological Propertes and Storage Stabilty of SEBS Polymer Modified Btumen”, International Journal of Engineering Science and Technology, Vol.5,No.05, (2013).

10-Al-Zubaidi, M. A., "Modification of the Rheological Properties of Beeje Asphalt Using Different Additives and the Field of Their Uses", M.SC. Thesis, University of Mosul, (2013).

11-Can, J., "Asphalt Modification with Used Lubricating Oil”, Canadian Journal of Civil Engineering, 35(2): 148-157, (2008).

12-Arslan, L., K. and Taque, E., " Treatment of Asphalt from Heet Area Using the Residues of Basic Oils", Diala, Jour., Vol. 39, (2008).

13-Huff, B. J. and Vallerga, B. A., " Characteristic and Performance of AsphaltRubber of Materials Containing a blend of Reclaimed and Crumb Rubber", Transp. Res. Rec.; Chem. Abs., (1982), 96, 22300u, (1981).

14-Al-Sayd Toohi, H. T. S., "Study of Rheological Properties of Modified Qiayarah Asphalt by Reclaim Tires Rubber Using Microwave Technique", M.Sc. Thesis, University of Mosul, (2013).

15-Sadeque, M., Patil, D.K.A., " Rheological Properties of Recycled Low Density Polyethlene and Polypropylene Modified Btumen", Internationol Journal of Advanced Technology in Civil Engineering, Vol.-2, Issue-2, (2013). 
16-Bulato, V., O., Rek,V. and Markovic, K.J., "Polymer Modified Bitumen", Materials Research Innovation Vol.16,No.1(2012).

17-Al-Liheby, S. A. S., "Effect of Some Polymeric Additives to the Asphalt Materials and the Field of Their Uses", M.Sc. Thesis, University of Mosul, (2012).

18- Anjan, S., Veeraragavan, A., "Construction and Building Materials", Vol. 24, Issue 11, pp: 2041-2100, (2010).

19-Salih, L. A., "Effect of Air Blowing on the Stability of Mixtures (Qayarah Asphalt-Sulpher), Al-Rafidden Science Journal, Vol. 29, No. 2, pp 174-190, (2008).

20-Ali, L. H., Al-Ghannam, K. A., "Investigation into Asphaltenes in Heavy Crude Oils", J. Fuel, Vol. 60, pp: 1043-1046, (1981)

21- ASTM, Part II, (D $36-70)$, P. 27, (1972).

22-ASTM, Section 4, (D-83), P. 97, (1986).

23-ASTM, Section 4, (D-85), P. 127, (1986).

24-Traxler, R. N., " Asphalt: Its Composition, Properties in Uses", Haff, Ltd., London, p. 3. 72-72, (1961).

25-Adam, Georgius A., "Industrial Chemistry", Dar Al-Kutub for Printing and Publishing, University of Mosul, pp. 168, 171, 440-443, (1986).

26- Ali, L. H. and Al-Dobouni, I. A. "Petroleum: Origin, Composition, and Structure", Mosul University, pp. 236, 237, 271, 272, 274, (1986).

27-Al-Frakh, Y. and Abu Shihada, A., "Production of Bitumen Grades", Brit., UK Pat. Appl., GB2, 028,406; Chem. Abs., (1982), 96, 126085g, (1981).

28-Holbsen, G. D., “ Modern Petroleum Technology”, $4^{\text {th }}$ ed., Ltd. Britain, pp: 804-806, 810-811, (1975).

29-ASTM (1969), Part 11, (D491-41), p. 250-251, (1969).

30-Central Organization for Standardization and Quality Control (COSQC), Standard Specification No. 1196 about the Asphalt used in Roofing Operations, Ministry of Construction and Housing and Municipalities and Public Works, Iraq, (1988).

31-State Commission for Roads and Bridges (SCRB), Department of Design and Study, Ministry of Construction and Housing and Municipalities and Public Works, Baghdad, (2001). 